\title{
Mandibular two implant- supported overdentures as the first choice standard of care for edentulous patients - the York Consensus Statement
}

Please send any ideas for feature articles for consideration to:

Rowena Milan,

British Dental Journal,

The Macmillan Building

4-6 Crinan Street

London

N1 9XW

Email:r.milan@nature.com

The Annual Conference of the BSSPD (British Society for the Study of Prosthetic Dentistry) was held in York on 6 and 7 April 2009. At the symposium on mandibular overdentures, presenters offered a synopsis of the research available on the efficacy of implant-supported mandibular overdentures in the edentulous mandible. Emphasis was given to both qualitative and quantitative research based on patient-centred outcomes of treatment. A draft consensus was circulated to all presenters and to the Council members of the BSSPD and to BSSPD members on the Society's website. The statement was modified in the light of their comments, audience feedback following the presentations and members' feedback. We hope that this consensus statement will be a useful guide for patients and clinicians and that it will act to stimulate wider debate. We also hope that it will prove useful to other patient and professional organisations and will inform discussions with providers of national healthcare and with independent funders.

\section{INTRODUCTION}

In the seven years since the publication of the McGill Consensus Statement in 2002, ${ }^{1}$ uptake by dentists of implant technology for complete denture wearers has been slow. To help to improve this situation, this statement has been developed after examining the currently available data and is released as a support and follow-up to the McGill Consensus Statement.

This report was jointly created by members of the BSSPD Council and the panel of presenters at the BSSPD Conference in York on 6 and 7 April 2009. It is based on the data presented at the conference, the wider available scientific data and the experience of the BSSPD members and participants at the conference. It represents a wholly independent report and is not a policy statement for any profit-making body or business and, as such, is presented in line with the mission of the BSSPD to advance patient care, education and research in fixed and removable prosthodontics.

\section{CONSENSUS STATEMENT}

It is fully recognised that the rate of tooth loss in most industrialised countries has declined rapidly over the last two decades. Nevertheless, as a result of the steady increase in the mean age of industrialised societies, and because the overall prevalence of tooth loss increases with age, the proportion of edentulous individuals in these societies will remain significant. For over a century, the accepted treatment for edentulous patients has been to provide maxillary and mandibular dentures that rest on the soft tissues of the alveolar ridges and are not directly connected to them. This has been the traditional standard of care as there have been no practical alternatives. For many patients, these conventional complete dentures have allowed them to eat, to speak and to function in the wider society more easily than they could without any prostheses. Despite this, it is well recognised that many struggle to use dentures, particularly the lower denture, because of mobility and discomfort, and these difficulties have been shown to be linked with social, psychological and functional disabilities, a situation which can deteriorate further as the ridges reduce in size over time.

Clinical studies over the last two decades have been undertaken to determine the benefits to patients from the use of mandibular implants to support the lower denture. This formed the basis of the McGill Consensus statement of 2002, and the current statement addresses these questions in light of contemporary research. The objective is to see if the benefits of this treatment modality should be regarded as large enough for this group to propose it, rather than conventional dentures, as the first treatment option of choice for edentulous patients.

There has been a very large body of work published that has established that the survival of titanium dental implants is very high, especially in the anterior mandible, and that the rate of bone loss in the edentulous jaws is greatly reduced when implants have been placed. Until the last decade, there was much less data available on the benefits of using these implants to support mandibular prostheses, particularly basing the decision-making process on patient-centred data.

\section{Patient-centred outcomes}

Conventional complete dentures are supported by the edentulous ridges and the mucosa that overlies them. There is close contact, but no direct attachment between the prosthesis and the ridges, and the prostheses are constructed to maximise any potential retentive forces whilst attempting to minimise those that displace them. In such an active, muscularly-controlled environment this is problematic, and many patients have difficulties adapting to their dentures, particularly the lower denture. Edentulism is also associated with a less 
healthy diet. Many patients report that they have to modify their food choices, especially when eating in a social environment, because of the limitations of their dentures. Evidence also suggests that if patients are challenged to eat a different range of foods, their current satisfaction with their conventional dentures is reduced. In a wider context, patients' social interactions can be negatively affected by conventional dentures. People report avoiding going out to eat, being self-conscious of the presence of others as they feel they may notice them moving in the mouth when talking, eating or laughing and because they may find it difficult to wear their dentures for a prolonged period of time.

A substantial body of evidence is now available demonstrating that patients' satisfaction and quality of life with implant-supported mandibular dentures is significantly greater than for conventional dentures. Much of this data comes from high quality randomised controlled trials. Patients report greater satisfaction with the stability and retention of their prostheses which, in turn, appears to contribute to greater satisfaction with comfort and ability to chew different foods. Patient's objective chewing efficiency is also significantly increased when the lower conventional denture is stabilised by means of implants.
There is accumulating evidence that these advantages can be carried into old age.

More recent evidence demonstrates that patients with mandibular implant-supported overdentures are more likely to positively modify their diet than patients with conventional dentures, particularly following dietary interventions. In contrast to conventional denture wearers, when encouraged to modify their diet, the satisfaction with their prosthesis of those wearing implant overdentures appears to increase.

\section{Cost}

This remains a very real perceived barrier to the delivery of implant supported prostheses. Whilst they are certainly more expensive than conventional dentures, the use of just two implants can keep the initial cost to a minimum. Furthermore, deferring the whole cost over the expected life span of the patient shows that the annual difference in costs between the two modalities is relatively small, especially compared with the initial first year costs.

\section{Conclusions}

With the advent of dental implants there is now more than one available treatment for edentulous patients. Current evidence suggests that the restoration of the edentulous mandible with a conventional denture is a much poorer alternative than the use of an implant-supported prosthesis. There is now a large body of evidence that supports the proposal that a two-implant supported mandibular overdenture should be the minimum offered to edentulous patients as a first choice of treatment.

J. Mark Thomason, ${ }^{*}$ United Kingdom; Jocelyne Feine, Canada; Catherine Exley, United Kingdom; Paula Moynihan, United Kingdom; Frauke Müller, Switzerland; Ignace Naert, Belgium; Janice S. Ellis, United Kingdom; Craig Barclay, United Kingdom; Chris Butterworth, United Kingdom; Brendan Scott, United Kingdom; Christopher Lynch, United Kingdom; Dominic Stewardson, United Kingdom; Philip Smith, United Kingdom; Richard

Welfare, United Kingdom; Paul Hyde, United Kingdom; Robert McAndrew, United Kingdom; Michael Fenlon, United Kingdom; Stewart Barclay, United Kingdom; Dean Barker, United Kingdom

*Correspondence to: J. Mark Thomason, Professor of Prosthodontics and Oral Rehabilitation, Newcastle University School of Dental Sciences

Email: J.M.Thomason@ncl.ac.uk

1. Feine J S, Carlsson G E, Awad M A et al. The McGill Consensus Statement on Overdentures. Montreal, Quebec, Canada. May 24-25, 2002. Int J Prosthodont 2002; 15: 413-414.

DOI: 10.1038/sj.bdj.2009.728 